Asian Journal of Engineering and Applied Technology

ISSN 2249-068X Vol. 7 No. S2, 2018, pp.87-92

(C) The Research Publication, www.trp.org.in

\title{
Effect of Rotary Tool on EDM Performance
}

\author{
Manpreet Singh Sidhu ${ }^{1}$ and Sukhpal Singh Chatha ${ }^{2}$ \\ ${ }^{1}$ Department of Mechanical Engineering, GNDEC, Ludhiana, Punjab, India \\ ${ }^{2}$ Yadavindra College of Engineering, Punjabi University Guru Kashi Campus, Talwandi Sabo, Bathinda, Punjab, India \\ E- Mail: 767.manpreet@gmail.com
}

\begin{abstract}
Machining is a broad term which is used to describe removal of material from a work-piece. The rotary electrode discharge machining (EDM) has emerged as a successful technique to remove the material from the work surface of different materials such as tool steel, ceramic, metal matrix composite and inconel etc. In this study, the machining characteristics of the die steel of $\mathrm{H}-13$ by using rotary electrode discharge machining (EDM) with a cylindrical electrode were investigated. Magnetic field assisted EDM has also been examined on the machining parameters (MRR, TWR and SR). From the results it is observed that rotary tool EDM showed the better results with regards to MRR and SR. The rotary electrode improves the machining performances due to better flushing of debris from the machining gap.
\end{abstract}

Keywords: Rotary Electrical Discharge Machining, MRR, TWR and Surface Roughness

\section{INTRODUCTION}

Machining is a broad term which is used to describe removal of material from a work-piece. Materials such as tool steel, ceramic, metal matrix composite and inconel are used most commonly in die moulding, aerospace, surgical and automotive industries. Machining of these materials by using of traditional machining is difficult due to high wear and corrosion resistance, hardness and toughness properties [1]. The non conventional machining methods, EDM is used extensively to machine difficult-to-machine materials. The spark erosion process or EDM is a relatively modern machining process having distinct advantages over other machining processes and so its use is getting more and more widespread. During EDM it is not possible to obtain the high material removal rate with low surface roughness and tool wear. Therefore, it becomes important to analyze and optimize EDM parameters to achieve the highest material removal rate and at the same time minimum surface roughness and tool wear. The parameters of EDM which affects these responses are current, voltage, pulse on time (T-on), pulse off time (T-off), electrode material, dielectric used and operator skill etc. EDM does not make direct contact between the electrode and the work-piece, where it can eliminate mechanical stresses chatter and vibration problems during machining [2].

Flushing the debris from the machining gap is one of the major challenges in EDM process because it affects the material removal rate and surface roughness. By considering of these reasons, researchers focus on the methods which improve the flushing in EDM.Various

methods are used to clear the debris from the maching gap like side flushing, force suction flushing etc. but force suction flushing is not feasible compare to other methods. The rotating electrode compared with the stationary electrode various performance measures. By improving the surface of the machining gap due to the better flushing action the better results are achieved like high MRR with little tool wear[3].Combination of high frequency vibration and rotation of the electrode is also found to be effective in attaining a high MRR at specific surface roughness [4]. The rotational effect of cylindrical (pin shaped) or disc shaped electrode tool is measured in revolution/minute. The rotational movement of electrode is normal to the work surface and with increasing the speed a centrifugal force is generated, which causes debris to remove faster from the machining zone [5]. Fig. 1, Shows the effect of rotary tool on the performance of EDM.

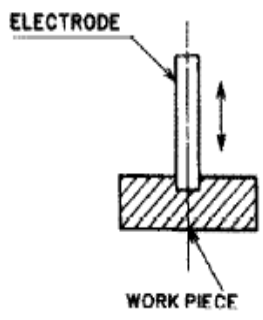

(a)

Fig. 1 (a) Stationary tool (b) Rotating tool (c) Orbital rotating tool [3]

The centrifugal force generated throws a layer of dielectric in to the machining gap, induces an atmosphere for better surface finish, prevent arching and improves MRR [6]. Rotary electrical discharge machining in induced magnetic field produced higher material removal rate and decreases electrode wear rate as compared with machining in a nonmagnetic field. Thus, introduction of magnetic field increases the productivity of this process [7]. In EDM process, the magnetic force expelled the debris away from machining zone and improves the characteristics of this operation [8].

As per the literature review, the flushing debris on machining gap is the major problem in EDM and its effect on the surface roughness (SR), material removal rate (MRR) and tool wear rate (TWR). To investigate these factors, various researchers had worked on it but out of those only 
few had worked on EDM by using the rotary tool along with the magnetic field. So lot of work can be done in this area to increase the efficiency of electro discharge machine. With an aim to improve flushing of debris and to improve the MRR, SR, combination of rotary tool along with the magnetic field will be used in EDM in the present study.

\section{EXPERIMENTAL DETAILS}

\section{A. Experiment Setup}

The experimentation was conducted using the automatic ELEKTRAPLUS PS 20 (T-3822) machine as shown in Fig. 2 . This machine is internally computer controlled, working with the automatic panel by entering the defined parameter values. In an existing EDM machine, tool holder was stationary and a special rotary head has been attached to the quill of the EDM machine to provide rotary motion to the electrode. The electrode-rotating device consists of a rotary tool and a variable speed control unit with a magnetic field around the tool-work piece system. The schematic representation of rotary tool set up is shown in Fig. 2.

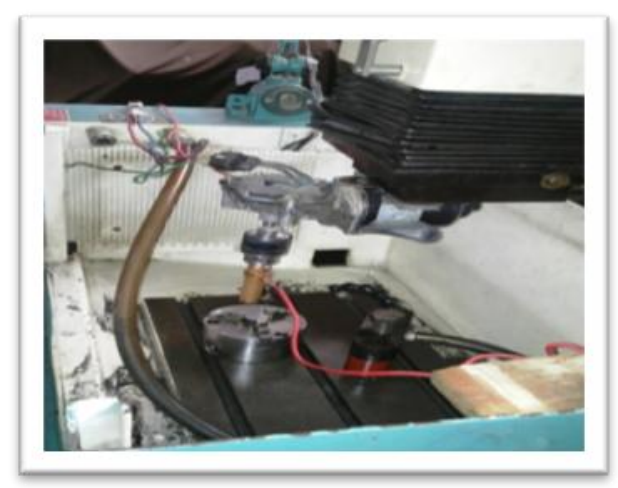

Fig. 2 Experimental setup: The automatic ELEKTRAPLUS PS 20 (T-3822) machine with mechanism of the rotational tool

\section{B. Experiment Materials}

In this study, the die steel H-13 of circular cross-section bar was cut into the specimen of $20 \mathrm{~mm}$ diameter and $10 \mathrm{~mm}$ length to carry out the perform of EDMed operations and then faced and ground with grinding wheel. The cylindrical pure red colour of copper (99.9\% purity) with $16 \mathrm{~mm}$ diameter was selected for electrode according to the design of rotary fixture. The dielectric fluid (kerosene) is flushed to the machining gap by side flushing method. The chemical composition (wt.\%) of H-13 die steel shown in Table 1.

TABle I Chemical Composition (WT.\%) OF H-13 Die SteEL

\begin{tabular}{|l|l|l|l|l|l|l|l|l|}
\hline \multicolumn{7}{|c|}{ Chemical composition (wt. \%) } \\
\hline $\begin{array}{l}\text { Allo } \\
\text { y }\end{array}$ & $\mathrm{C}$ & Mn & $\mathrm{S}$ & $\mathrm{P}$ & $\mathrm{Si}$ & $\mathrm{Cr}$ & $\mathrm{Mo}$ & $\mathrm{V}$ \\
\hline $\mathrm{H}-13$ & 0.30 & 0.20 & 0.0 & 0.0 & 0.80 & 4.75 & 1.25 & 0.50 \\
& - & - & 5 & 5 & - & - & - & - \\
& 0.40 & 0.40 & & & 1.20 & 5.50 & 1.75 & 1.20 \\
\hline
\end{tabular}

\section{Experimental Procedure}

In this work, the experimental procedure has two stages. In the first stage, the effect of process parameters on EDM performance was investigated while the electrode is stationary, with or without the magnetic field on machining zone. In the second stage, the effects of rotational speed of electrode on machining performance were investigated with or without the magnetic field on machining zone. Variable parameters of this investigation were current (I), pulse on time (Ton), the tool rotation and magnetic field. The process parameters were selected which affects the performance of EDM are used in the present experimentation are shown in Table II with their range.

TABle II PRocess PARAmeters AND TheIR RANGE

\begin{tabular}{|c|l|c|c|c|}
\hline S. No. & Variable Parameters & \multicolumn{3}{|c|}{ Values } \\
\hline 1. & Current $(\mathrm{A})$ & 4 & 6 & 8 \\
\hline 2. & T-on $(\mu \mathrm{s})$ & 50 & 100 & 150 \\
\hline 3. & Tool Rotation $(\mathrm{rpm})$ & 0 & 32 & 54 \\
\hline 4. & Magnetic Field $(\mathrm{T})$ & \multicolumn{2}{|c|}{1} & \multicolumn{2}{|c|}{0} \\
\hline
\end{tabular}

In the above table, the magnetic field column has two variables one is with and second is without magnetic field is coded as 1 and 0 respectively. According to the Taguchi quality design concept, a L18 orthogonal arrays table with 18 rows (corresponding to the number of experiments) was chosen for the experiments. In order to decrease the experimental error, two specimens have been tested for each kind of conditions. Before experimentation, the workpiece top and bottom faces were ground to a good surface finish using a magnetic surface grinding machine. The bottom of the tool is polished using a very fine grade emery sheet before every experiment. The initial-weight of the workpiece and tool was weighed using a digital weighing machine. The workpiece and tool were connected to the negative and positive terminals of power supply, respectively. The dielectric fluid was flushed at a pressure of $0.5 \mathrm{kgf} / \mathrm{cm}^{2}$ from side of the electrode.

Tool generates the mirror image profile on the work piece. The experiments were conducted in a random order so as to remove the effects of any unaccounted factors. End of each experiment, the work- piece and tool was removed from the machine, washed, dried, and weighed on an electronic balance. The machining time was $20 \mathrm{~min}$ for all experiments and therefore the machining depth is not constant for all experiments and depends on MRR. The roughness of surface was measured four times stochastically and average of the data was reported as Ra value. Then find out the MRR and TWR and the surface roughness $(\mathrm{Ra})$ of the work piece has been measured using the roughness tester. After observed the experimentation data from the test, the data is summarized to find the optimal values from the parameters being taken in this investigation. Taguchi method cannot judge and find the effect of individual 
parameters on the entire process while percentage contribution of individual parameters can be well determined using ANOVA in the MINITAB 17 program.

\section{DISCUSSION}

\section{A. Material Removal Rate (MRR)}

After the experiments have been done in two stages then find out the signal to noise ratios of MRR. The Fig. 3 shows the Main effects plot for $\mathrm{SN}$ ratios and Table III shows response table of $\mathrm{SN}$ ratios of MRR.

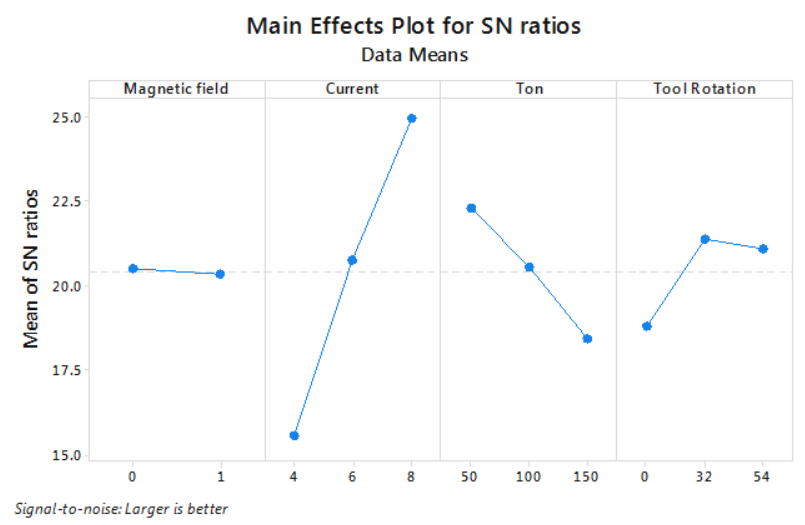

Fig. 3 Main effects plot for SN ratios (MRR)

TABLE III RESPonse TABLE Of Signal To Noise Ratio

\begin{tabular}{|c|c|c|c|c|}
\hline Level & Magnetic field & Current & Ton & Tool Rotation \\
\hline 1 & 20.51 & 15.56 & 22.31 & 18.81 \\
\hline 2 & 20.34 & 20.77 & 20.55 & 21.40 \\
\hline 3 & & 24.96 & 18.42 & 21.07 \\
\hline Delta & 0.17 & 9.41 & 3.89 & 2.59 \\
\hline Rank & 4 & 1 & 2 & 3 \\
\hline \multicolumn{5}{|c|}{ *Larger is better } \\
\hline
\end{tabular}

\section{Effect of magnetic field on MRR}

The Fig. 3 shows that the MRR is decreased with the magnetic field in EDM process, the intensity of electrical field which exist between two electrodes depend on differential of voltage and the distance between two electrodes. When a magnetic field is established vertically around the electrical field, the path of ion migration between two electrodes will be changed from linear to cycloid motion due to Lorentz force. Therefore the curvature of ion migration increases by applying the magnetic field and the ions are not focused well in the spaces between electrodes and lead to reduction in the MRR. But without the magnetic field this curvature is less than with one and leads to better concentration of ions in machining gap and improves the MRR.

It is well known that force acting due to induced magnetic field is proportional to magnetic field strength and mass of the eroded particles. Here, magnetic force was not able to remove eroded particles from the gap, as their mass was low. Thus, left over eroded particles created short circuits, resulting in reduced MRR with magnetic as compare without magnetic field on machining zone [7].

\section{Effect of Current on MRR}

Fig. 3 shows the relationship between MRR and different peak current values. The figure shows that the MRR increases with the increase in current. It indicates that the MRR varies almost linearly with the current. At pulse on time $50 \mu \mathrm{s}, \mathrm{MRR}$ increases as current increases from $4 \mathrm{~A}$ to $8 \mathrm{~A}$. MRR increased with an increase in discharge current (Id) due to the spark energy. Spark energy increases with current which leads to higher crater volumes. Thus MRR increases with current [14].

\section{Effect of Pulse on time (Ton) on MRR}

It can be observed from the figure that the MRR decreases with the increase in pulse on time (Ton). The highest MRR is achieved at low pulse on time $(50 \mu \mathrm{s})$. As the experimentation results shows that least MRR at high pulse on time this may be due to that as the pulse on time increases, the dielectric medium which was injected through the machining zone was insufficient to remove the debris from the machining gap. Due to insufficient of removal of debris from the machine surface, the melting material again solidify on the same work surface and make the hard layer between the gap of work surface and electrode. Thus, left over eroded particles created short circuits, which intern results in decrease of MRR.

\section{Effect of Tool rotation on MRR}

Fig. 3 shows the MRR versus tool rotational speed in three stages $0 \mathrm{rpm}, 32 \mathrm{rpm}$ (Low value) and $54 \mathrm{rpm}$ (High value). The results shows that in absence of magnetic field, the MRR increases as the rotary tool speed increases from the 0 to low value. The increase in MRR with rotary tool is attributed to the better flushing on machining zone. The rotary tool imparts a whirl and effectively flushes the gap and prevents arcing. MRR is reduced from low value to high value gradually. This is due to that in high values of tool speed the turbulence is increased and it causes to damp the plasma channel or this may be due to non-efficient removal of eroded particles from the gap and which interns the MRR [1,7].

The above mean of $\mathrm{S} / \mathrm{N}$ ratio (Fig. 3) plot represents the relation between the MRR with respective selected parameters which gives the optimal parameters (Magnetic field $=0$, Current $=8 \mathrm{~A}$, Ton $=50 \mu \mathrm{s}$ and Tool rotation $=32 \mathrm{rpm}$ ) as a final result.

\section{B. Tool Wear Rate (TWR)}

The tool wear rate is the volume of material removed per unit time $\left(\mathrm{mm}^{3} / \mathrm{min}\right.$.) from the electrode by a series of 
rapidly recurring current discharges between the electrodes separated by a dielectric fluid \& subject to an electric discharge. The TWR has been calculated by using the work piece loss from the work piece divided by density and machining time (Singh et al., 2011). Table 3.1 shows response table of $\mathrm{SN}$ ratios of TWR.

TABle Iv Response TABle For Signal To NoIse Ratios

\begin{tabular}{|c|c|c|c|c|}
\hline Level & Magnetic field & Current & Ton & Tool Rotation \\
\hline 1 & 5.3180 & 13.2158 & 0.4015 & 17.3088 \\
\hline 2 & 7.4316 & 5.1501 & 5.5183 & 1.7534 \\
\hline 3 & & 0.7584 & 13.2046 & 0.0621 \\
\hline Delta & 2.1136 & 12.4575 & 12.8031 & 17.2467 \\
\hline Rank & 4 & 3 & 2 & 1 \\
\hline
\end{tabular}

*Smaller is better

The Fig. 4 shows the Main effects plot for SN ratios.

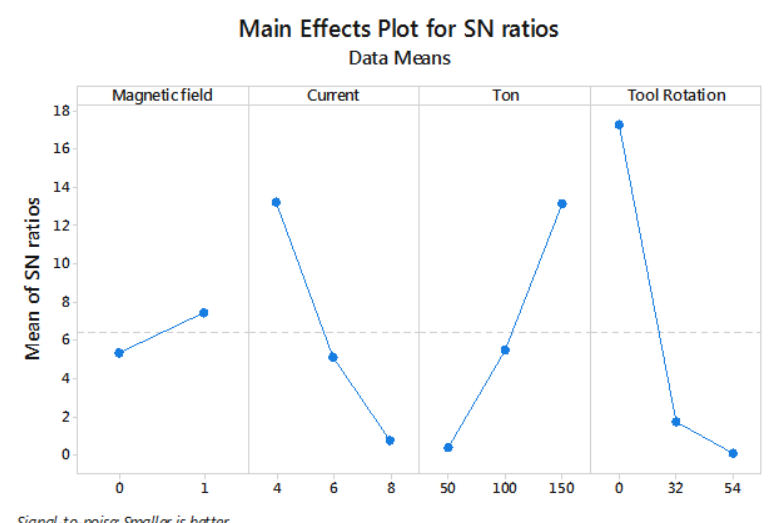

Fig. 4 Main effects plot for SN ratios (TWR)

\section{Effect of magnetic field on TWR}

The TWR tends to decreased with the effect of magnetic field (as compare with the conventional machining) on the machining zone. When a magnetic field is established vertically around the electrical field, the path of ion migration between two electrodes will be changed from linear to cycloid motion due to Lorentz force [1]. Therefore the curvature of ion migration increases by applying the magnetic field and the ions are not focused well in the spaces between electrodes and lead to reduction in the MRR as well as TWR.

\section{Effect of current on TWR}

Fig. 4 shows the relationship between TWR and at different peak current. It can be observed from the figure that the TWR increases with the increase in current. The Fig. 4 explains that the TWR varies almost linearly with the current. TWR was found to increase with an increase in discharge current (Id). Spark energy increases with current which leads to higher crater volumes from the electrodes by the action of vaporizing, melting in the the discharge gap which interns TWR as well as MRR increases with current [15].

\section{Effect of pulse on time (Ton) on TWR}

Fig. 4 shows the relationship between TWR and at different values of pulse on time (Ton). It can be shown from the figure that the TWR decreases with the increase in pulse on time (Ton) from $50 \mu \mathrm{s}$ to $150 \mu \mathrm{s}$. As the experimentation results shows that least TWR is observed at pulse on time $150 \mu \mathrm{s}$. The higher crater volumes are produced at higher energy (Ton 150 $\mu$ s) but MRR as well as TWR is decreased. As the pulse on time increases, the dielectric medium which was injected through the machining zone was insufficient to remove the eroded particles from the machining gap. Due to non-sufficient of removal of eroded particles from the machine surface, the melting material again solidify on the same work surface and make the hard layer between the gap of work surface and electrode. Thus, left over eroded particles created short circuits, which interns the MRR as well as TWR decreases.

\section{Effect of tool rotation on TWR}

Fig. 4 shows the relationship between TWR and at different speeds of tool rotation. It can be observed from the figure that the TWR increases with the increase the tool speed from 0 to $54 \mathrm{rpm}$ due to its centrifugal action by rotating tool. A new layer of dielectric fluid is thrown into the machining gap, which imparts the effective flushing on machining zone. This induces a conductive atmosphere for effective discharge, which in turn results in increase of MRR and TWR [4].

The above mean of $\mathrm{S} / \mathrm{N}$ ratio (Fig. 4) plot represents the relation between the TWR with respective to selected parameters, which gives the optimal parameters (Magnetic field $=01$, Current $=4 \mathrm{~A}$, Ton $=150 \mu \mathrm{s}$ and Tool rotation=0) as a final result.

\section{Surface Roughness (SR or Ra)}

After the experiments have been done in two stages then find out the signal to noise ratios of SR. The Fig. 5 shows the Main effects plot for $\mathrm{SN}$ ratios and Table 5 shows response table of $\mathrm{SN}$ ratios of SR.

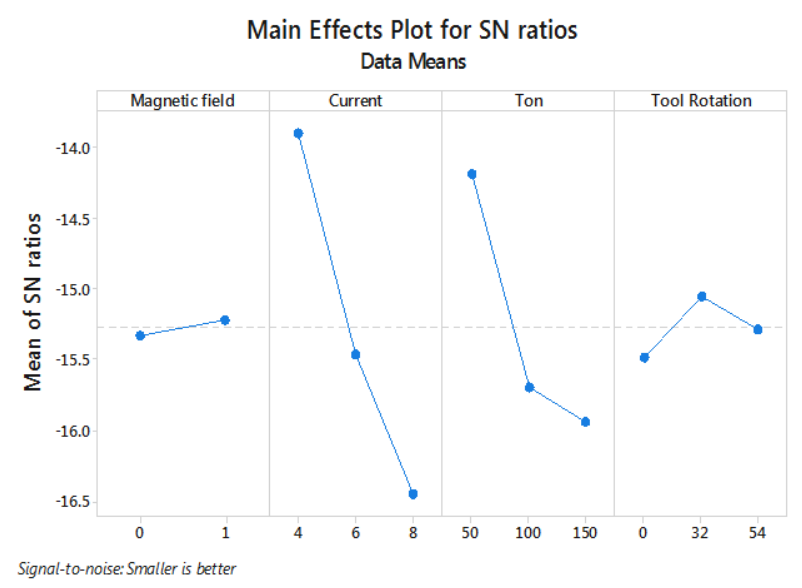

Fig. 5 Main effects plot for $\mathrm{SN}$ ratios $(\mathrm{Ra})$ 
TABLE V RESPONSE TABLE For Signal To NOISE RATIOS

\begin{tabular}{|c|c|c|c|c|}
\hline Level & $\begin{array}{c}\text { Magnetic } \\
\text { field }\end{array}$ & Current & Ton & $\begin{array}{c}\text { Tool } \\
\text { Rotation }\end{array}$ \\
\hline 1 & -15.33 & -13.89 & -14.18 & -15.49 \\
\hline 2 & -15.21 & -15.46 & -15.70 & -15.04 \\
\hline 3 & & -16.45 & -15.93 & -15.28 \\
\hline Delta & 0.12 & 2.56 & 1.76 & 0.44 \\
\hline Rank & 4 & 1 & 2 & 3 \\
\hline
\end{tabular}

\section{Effect of magnetic field on surface roughness}

From the Fig.5, it is observed that surface roughness improved with the use of magnetic field in EDM. The use of magnetic field results in expelling the debris from the machining zone easily and quickly, which interns results in improvement of surface. As observed by Teimouri [1], when magnetic field applied on machining gap, the debris which were expelled from the machining gap remove the sharp edges from the machined surface. This results in better surface finish. Therefore, the surface roughness of the machined surface was fine in case of magnetic force assisted of the EDM process.

\section{Effect of current on surface roughness}

The Fig.5 depict significant effect of peak current on the surface roughness. It was observed that increase in current resulted in an increase in surface roughness value. When the discharge current is high, then the spark intensity and discharge power are more, subsequently causing a larger crater depth on the surface of the work piece, which resulted in high surface roughness value [16]. The SR increases by increasing the discharge energy. This is due to larger crater which is generated on the workpiece surface when the process moves to high energy level [1]. By increase in discharge current resulted in an increase in $R$ a value [6]. In this experimentation study, the fine surface is achieved at low current $(4 \mathrm{~A})$.

\section{Effect of T-on on surface roughness}

The Fig.5 shows the effect of pulse duration on surface roughness under peak current. The surface roughness value increased with increase in pulse duration. This is due to the fact that long pulse duration in the machining process may expand the plasma channel and decrease the energy density. Eventually, the long pulse duration produces a shallow crater on the surface of the work piece.

Ra value increases with an increase in Ton. For a higher Ton, deeper discharge craters are formed and more material is removed per spark since spark energy is directly proportional to Ton. Saha [14] also observed similar results of increase in SR with the increase of Ton. The fine surface is achieved at Ton $50 \mu$ s in the present work.

\section{Effect of Tool Rotation on Surface Roughness}

Fig. 5 shows the effect of tool rotational speed on SR at different parameters. It indicates that the surface roughness decreases to a certain minimum value and then increases with the use of rotating tool instead of the stationary tool as in the conventional machining. The least surface roughness is achieved at tool rotation speed 32rpm. When the rotary speed increases, due to the centrifugal force, it can expel the debris from machining gap and prevents the debris to adherence to workpiece surface at the next discharges. This whirl condition caused by rotary motion of the tool improves the surface quality and reduces the SR. But this effect is saturated at higher speeds, and beyond the 32rpm the SR gradually increases [1].

The above mean of $\mathrm{S} / \mathrm{N}$ ratio (Fig. 5) plot represents the relation between the Ra with respective selected parameters which gives the optimal parameters (Magnetic field $=01$, Current $=4 \mathrm{~A}$, Ton $=50 \mu \mathrm{s}$ and Tool rotation $=32 \mathrm{rpm}$ ) as a final result.

\section{CONCLUSION}

Main conclusions of this present work can be summarized as follows:

1. Rotary tool with magnetic field improves the machining performance. MRR, TWR and surface finish increases by using rotary tool electrode as compared to stationary tool. Rotary tool electrode apply a centrifugal force and whirl condition which can remove the debris from the machining gap and resulting in better flushing condition and improves the MRR, TWR and surface finish.

2. Surface finishing improved by applying magnetic field on the machining zone because magnetic field can expel the debris from machining gap. So magnetic field improves the surface quality.

3. With the increase of pulse on time from $50 \mu$ s to 150 $\mu \mathrm{s}$, the MRR and TWR decreased, which may be due to the inefficient flushing of debris by machining.

4. With the increase of value of current $4 \mathrm{~A}$ to $8 \mathrm{~A}$, the MRR improved however surface roughness increased. The higher value of MRR at higher value of current may be attributed to the input of high discharge energy, which intern results in deep craters, which results higher MRR and poor surface finish.

\section{REFERENCES}

[1] J.R. Crookall, C.J. Heuvelman, "Electro-discharge machining-the state of the art", Annual of the CIRP, Vol. 20, pp. 113-120, 1971.

[2] R. Ramaswami, S. Louis raj, "A study of wear and surface finish during spark erosion machining of high speed tool steel", $J$ Wear, Vol. 24, pp. 153-160, 1973.

[3] K.P. Rajurkar, Edited by Richard, C. Dorf and Andrew Kusiak, "Nontraditional Manufacturing Processes, Handbook of Design, Manufacturing and Automation", John Wiley and Sons, USA.1994.

[4] J.S. Soni, G. Chakraverti, "Machining characteristics of titanium with rotary electro discharge machining", $J$ Wear, Vol. 171, pp. 51-58, 1994. 
[5] Y.S. Wong, L.C. Lim,; L.C. Lee, "Effect of Flushing on Electro Discharge Machined Surface", J Mater Process Tech Vol. 48, pp. 299-305, 1995.

[6] J.S. Soni,; G. Chakraverti, "Performance evaluation of rotary EDM by experimental design technique", J Defense Sci , Vol. 47, pp. 6573, 1997.

[7] W.H. Yang, Y.S. Tarng, "Design optimization of cutting parameters for turning operations based on the Taguchi method", $J$ Mater Process Tech Vol. 84, pp. 122-129, 1998.

[8] C. Sommer, "Non-traditional machining handbook", First edition, Advance Publishing, Houston, 2000.

[9] M. Ghoreishi, J. Atkinson, "A comparative experimental study of machining characteristics in vibratory, rotary and vibro-rotary electro-discharge machining", J Mater Process Tech, Vol. 120, pp. 374-384, 2002.

[10] T.R. Lin, "Optimization technique for face milling stainless steel with multiple performance characteristics", Int J Adv Manuf Tech, Vol. 19, p. 330-335, 2002.

[11] H.T. Lee, T.Y. Tai, "Relationship between EDM parameters and surface crack formation", J Mater Process Tech, Vol. 142, pp. 676683, 2003.

[12] K.H. Ho, S.T. Newman, "State of the art electrical discharge machining (EDM)", Int J Mach Tool Manu 43, pp. 1287-1300, 2003.

[13] S. Shaji, V. Radhakrishnan, "Analysis of process parameters in surface grinding with graphite as lubricant based on the Taguchi method", J Mater Process Tech, Vol. 141, pp. 51-59, 2003.

[14] B. Mohan, A.Rajadurai, K.G. Satyanarayana, "Electric discharge maching of $\mathrm{Al}-\mathrm{SiC}$ metal matrix composites using rotary tube electrode", J Mater Process Tech, pp. 978-985, 2004.

[15] Ghosh, Amitabha and Malik, Ashok Kumar, "Manufacturing Science", East West Press, New Delhi, pp. 383-403, 2005.

[16] P.C. Pandey, H.S. Shan, "Modern Machining Processes", Tata McGraw-Hill, New Delhi, 2005, pp. 86-114, 2005.

[17] Wang, Yan. Hu, Dejin., "Study on the inner surface finishing of tubing by magnetic abrasive finishing", Int J Mach Tool Manu, Vol. 45, pp. 43-49, 2005.

[18] N.M. Abbas, D.G. Solomon, Bahari, Md.F., "A review on current research trends in electrical discharge machining (EDM)", Int J Mach Tool Мапи, Vol. 47, pp. 1214-1228, 2007.
[19] Aggarwal, Aman. Singh, Hari. Kumar, Pradeep. Singh, Manmohan, "Optimizing power consumption for CNC turned parts using response surface methodology and Taguchi's technique-A comparative analysis", J Mater Process Tech Vol. 20, pp. 373-384, 2008.

[20] C.H. Che Haron, J.A. Ghani, Y. Burhanuddin, Y.K. Seong, C.Y. Swee, "Copper and graphite electrodes performance in electricaldischarge machining of XW42 tool steel" J Mater Process Tech, Vol. 201, pp. 570-573, 2008.

[21] K. D. Chattopadhyay, P. S. Satsangi, S. Verma, P. C. Sharma, "Analysis of rotary electrical discharge machining characteristics in reversal magnetic field for copper-en8 steel system", Int J Adv Manuf Tech, Vol. 38, pp. 925-937, 2008.

[22] P. Kuppan, A. Rajadurai, S. Narayanan, "Influence of EDM process parameters in deep hole drilling of Inconel 718", Vol. 38, pp. 74-84, 2008.

[23] Lin, Yan-Cherng. Lee, Ho-Shiun., "Machining characteristics of magnetic force-assisted EDM", Int J Mach Tool Manu, Vol. 48, pp. 1179- 1186, 2008.

[24] G.S. Prihandana, M. Mahardika, M. Hamdi, Y.S. Wong, K. Mitsui, "Effect of micro-powder suspension and ultra sonic vibration of dielectric fluid in micro-EDM processes-Taguchi approach", Int $J$ Mach Tool Manu, Vol. 49, pp. 1035-1041, 2009.

[25] S.K. Saha, S.K. Choudhury, "Experimental investigation and empirical modeling of the dry electric discharge machining process" Int J Mach Tool Manu 49, pp. 297-308, 2009.

[26] A. Pandey, S. Singh, "Current research trends in variants of Electrical Discharge Machining: A review", Int J Eng Sci and Tech Vol. 2, No. 6, pp. 2172-2191, 2010.

[27] M. Kuniedas, A. Kameyama,"Study on decreasing tool wear in EDM due to arc spots sliding on electrodes", Precis Eng, Vol. 34, pp. 546-553, 2010.

[28] Teimouri, R. Baseri, H., "Effects of magnetic field and rotary tool on EDM performance", J Manuf Process, Vol. 14, pp. 316-322, 2012.

[29] Yuna Yahagi, Tomohiro Koyano, Masanori Kunieda, and Xiaodong Yang (2012), "Micro Drilling EDM with High Rotation Speed of Tool Electrode Using the Electrostatic Induction Feeding Method", Procedia CIRP, Vol. 1, pp. 162 - 165, 2012.

[30] B. Ekmekci, A. Sayar, "Debris and Consequences in Micro Electric Discharge Machining of Micro-holes", Int J Mach Tool Manu, Vol. 65, pp. 58-67, 2013. 